\title{
The MPI 2.2 Standard and the Emerging MPI 3 Standard
}

\author{
Richard L. Graham \\ Oak Ridge National Laboratory, \\ USA
}

The Message Passing Interface (MPI) 2.1 standard (http://www.mpi-forum.org) has served the parallel technical and scientific applications community well over the last decade, and has become the ubiquitous communications application programming interface for this community. However, this extensive use has also exposed areas where this standard can be strengthened to meet the challenges of emerging ultra-scale computing. This talk will describe the changes introduced to the standard in latest version of the MPI standard, 2.2, and current work targeting version 3 of this standard.

The latest version of the MPI standard, version 2.2, is the culmination of a year and a half effort to modernize the standard. This includes updating the support for Fortran (2003) and C (99) language specifications, consolidating the standard into a single document, and many textual changes to correct errors, and clarify the standard.

Work on MPI 3 is aiming to modify the standard to better meet the needs of the emerging ultra-scale computer systems, and is expected to introduce large changes to the standard. The addition of support for nonblocking collectives to this standard has already won preliminary approval from the forum, and is awaiting final approval of the full MPI 3 standard. The other areas gaining a lot of attention include (1) adding support for MPI fault tolerance, (2) improved support for remote direct memory access, and (3) better standard support for debugging MPI based applications and extracting performance characteristics of such libraries. 